\title{
Homöopathie und Psychologie
}

\author{
Wenn Hahnemann und Freud sich heute treffen würden
}

Von Petra-Bindia Schmieder

\begin{abstract}
- Zusammenfassung
Das heutige Wissen und die Erkenntnisse aus der Psychologie entkräften wesentliche homöopathische Grundannahmen zur Entstehung und Bewertung von psychischen Erkrankungen. Besonders die psychodynamische Entwicklungstheorie und die Erkenntnisse aus der Neurologie und Traumaforschung können für ein tieferes Verständnis und eine Neuordnung wegweisend sein. Homöopathische Arzneien können psychische Entlastung herbeiführen, ersetzen aber keine Psychotherapie, weil zur Heilung auch Erkenntnis notwendig ist. Homöopathie und Psychotherapie sind kein Gegensatz, sondern eine Ergänzung.
\end{abstract}

- Schlüsselwörter

Psychologie, Psychotherapie, Tiefenpsychologie.

\section{- Summary}

The today's knowledge and the psychological understanding disprove essential basic assumptions in homeopathy about the origin and assessment of psychic diseases. Particularly the theory of psychodynamic development and the knowledge from neurology and trauma research can show the way to a deeper understanding and a reorganization. Homeopathic remedies can lead to psychic discharge, but do not substitute psychotherapy because for the healing process knowledge is necessary, too. Homeopathy and psychotherapy are no contrast, but a supplement.

\section{- Keywords}

Psychology, psychotherapy, depth psychology.
Ganzheitliche, den emotionalen Aspekt einbeziehende Sichtweisen und therapeutische Ansätze haben heute Hochkonjunktur. Ihnen traut man am ehesten zu, heilend zu wirken. In der Homöopathie ringen seit Jahrzehnten namhafte Homöopathen darum, Gemütssymptome in homöopathischen Arzneien zu systematisieren. Es existieren zahlreiche Strategien, wie die Gemütssymptome der Arznei im Patienten gefunden und übersetzt werden können.

Unverständlich bleibt dabei, warum Homöopathen sich der diagnostischen Mittel moderner Medizin bedienen, die heute zur Verfügung stehenden diagnostischen Mittel der Psychologie aber eher wenig nutzen. Vor allem die Erkenntnisse der psychodynamischen Entwicklungstheorie im Zusammenhang mit dem Wissen der Neurobiologie und der Traumaforschung sind für die Bewertung von psychischen Symptomen in der Homöopathie relevant. Sie entkräften wesentliche homöopathische Grundannahmen der Entstehung und Bewertung von psychischen Erkrankungen, eröffnen aber auch Möglichkeiten homöopathischer Behandlung.

Bestehende Grundannahmen:

- Gemütserkrankungen sind hereditär,

•. Gemütserkrankungen stellen ein Palliativum für schwere körperliche Krankheiten dar, Verschiebung von der körperlichen auf die seelisch/geistige Ebene,

- Gemütserkrankungen sind einseitige Erkrankungen,

- Gemütserkrankungen sind Folge von Unterdrückung körperlicher Symptome,

- Gemütssymptome lassen sich miasmatisch erklären und deuten,

- homöopathische Arzneien ersetzen Psychotherapie.

\section{Geisteskrankheit aus der Sicht Hahnemanns}

Hahnemann lebte in einer Zeit, in der es so gut wie kein Verständnis für Geisteskrankheiten gab. Die damals übliche Behandlung kann man nur als Folter bezeichnen. Es gab damals keine Alternative.

Ein Zeitgenosse Hahnemanns war Jean Etienne Dominique Esquirol, ein französischer Psychiater. Auch ihm ging es wie Hahnemann darum, die Missstände in Psychiatrien, damals Irrenanstalten genannt, abzustellen, in dem er 1818 eine eigene Untersuchungskommission ernannte. Später gründete und leitete er eines der ersten privaten Sanatorien für psychisch Erkrankte. Er war einer der ersten Psychiater in der Erforschung und Behandlung von Geisteskrankheiten. Ob Hahnemann von dessen Arbeit wusste, ist unklar. [6]

Behandlung Klockenbrings. Für wie verwerflich Hahnemann den damaligen Umgang mit psychisch Kranken hielt, findet seinen Ausdruck im $\S 228$ des Organon und in der Anmerkung 177. Wie so oft stemmte er sich auch hier gegen eine gesellschaftlich verrohte Strömung im Umgang mit psychisch Kranken. Dem kann man nur zustimmen. Dass er sich, und vor allem seiner Familie, den manisch-depressiven Klockenbring zumutete, spricht für seinen Mut und sein Engagement. Stramonium wird sicherlich besänftigend in den akuten Zuständen gewirkt haben, eine Heilung wage ich allerdings $\mathrm{zu}$ bezweifeln. Allein ein respektvoller Umgang mit Familienanschluss und damit ,gute Bindung“ mögen hier lindernd gewirkt haben. Für bipolare Störungen (angenommene Diagnose Klockenbrings) sind mehrjährige Latenzzeiten möglich - Klockenbring starb 2 Jahre nach Beendigung der 
Behandlung. Wie seine weitere Entwicklung gewesen wäre, bleibt also ungewiss.

\section{Entstehung der Gemütserkrankungen}

Hahnemann erklärte sich die Entstehung von Gemütserkrankungen auf dreierlei Weise:

- somatogen,

- psychogen und

๑ geistiger Natur.

Somatogene Entstehung. Somatogen (§215 und 216 Organon) bedeutet: Aus einer körperlichen Erkrankung heraus entwickelt sich eine psychische Veränderung! Die Gemütserkrankung sah er als Palliativum für schwere körperliche Krankheiten an. Sie galt für ihn als homöopathisch heilbar.

Psychogene Entstehung. Psychogen (§ 225 Organon) bedeutet: Emotionale Auslöser („durch anhaltenden Kummer, Kränkung, Ärgerniß, Beleidigungen und große, häufige Veranlassungen zu Furcht und Schreck“) oder dauerhaft belastende Umstände führen zu einer psychischen Störung/Irritation. Je länger die Ursache einwirkt, desto wahrscheinlicher wird sich im weiteren Verlauf eine körperliche Manifestation entwickeln.

Die Gemütserkrankung psychogener Entstehung galt für Hahnemann ebenso als homöopathisch heilbar.

Dies entspricht am ehesten dem heutigen psychosomatischen und psychodynamischen Ansatz. Diesem Umstand maß er erstaunlicherweise nicht besonders viel Bedeutung bei, er spricht von „einigen wenigen Gemüths-Krankheiten“, die auf diese Art entstehen. Aus Hahnemanns Lebzeiten ist eine interessante Dokumentation über Gewaltverbrechen bekannt. In Frankreich wurde 1886 eine Studie über 36176 (!) aktenkundig gewordene Fälle von Vergewaltigung und Sittlichkeitsverbrechen an Kindern bis zum Alter von 15 Jahren im Zeitraum von 1827-1870 von Bernard in seinem Buch „Über Sittlichkeitsvergehen an jungen Mädchen“ veröffentlicht [5].

In Deutschland wird es nicht anders ausgesehen haben. Damals wie heute wird die Dunkelziffer viel höher gewesen sein. Es sind also keine marginalen Ursachen für primäre Geistesstörungen in dieser Zeit. Natürlich stellt sexuelle Gewalt nur einen Teil von traumatisierenden Ursachen dar.

Entstehung „geistiger Natur“. Die Ursachen „geistiger Natur“ wie innere Haltung, Wertvorstellungen, die „Brille“, durch die wir die Welt sehen, sind auch „psychogen“. Eine Unterscheidung ergibt keinen Sinn. Wertesysteme entstehen natürlich ebenso durch Erfahrungen, die wir gemacht haben, und durch das, was uns die wichtigsten Bezugspersonen vermittelt haben. Unsere Gefühlslage ist eine Mischung aus Gefühlen und Gedanken. Kognitive Psychotherapieansätze fokussieren auf diese Auswirkungen: „Wie du denkst, so fühlst du.“

Psychische Erkrankungen aus „geistiger Natur“ (§ 224 Organon) entstehen aus inneren Haltungen, Moral- und Wertvorstellungen wie „Erziehungsfehlern, schlimmer Angewöhnung, verderbter Moralität, Vernachlässigung des Geistes, Aberglauben oder Unwissenheit“. Hier sah Hahnemann kein Wirkungsfeld für die Homöopathie. Er sah dies bestätigt und geradezu „.... als Merkmal, dass durch verständigendes, gut meinendes Zureden, durch Trostgründe oder durch ernsthafte und vernünftige Vorstellungen dieselbe nachlassen und sich bessern“.

\section{Heutige Erkenntnisse: psychodynamische (tiefenpsychologische) Entwicklungstheorie}

In der psychodynamischen Entwicklungstheorie geht man davon aus, dass bestimmte psychische Ich-Fähigkeiten in festgelegten Reifungsphasen der Säuglings- und Kleinkindentwicklung angelegt und erlernt werden. Dieses Lernen geschieht über eine liebevolle und unterstützende Beziehung der Eltern oder wichtiger Bezugspersonen zum Kind und wird in der Umwelt erprobt und weiterentwickelt.

Ich-Fähigkeiten sind unter anderem, sich differenziert wahrnehmen können. Dazu gehört, den eigenen Körper bewusst zu spüren, Körperempfindungen (Kribbeln, Schmerzqualitäten etc.) und Gefühle unterscheiden zu können, eigene innere Beweggründe, Impulse zu verstehen und ein eigenes Identitätsgefühl zu entwi- ckeln. Wahrnehmen können, worin andere Menschen ähnlich oder anders sind. Es beinhaltet auch, sich von Kränkungen abzugrenzen, ein positives Selbstbild aufbauen und halten zu können, Vertrauen zu entwickeln und Ambivalenzen aushalten zu können. Fähigkeiten, die man auch zum Aufbau von Beziehungen benötigt. Diese können nur gelingen, wenn man Verantwortung für sich selbst übernimmt und dies in eine gesunde Balance mit der Empathie und Fürsorge für andere bringt.

Je nachdem, wie früh, dauerhaft und stark diese Entwicklungsschritte behindert werden (durch inkompetente Beelterung, Gewalterlebnisse, Verwahrlosung, Kriegsfolgen etc.), desto weniger soziale Kompetenzen und Fähigkeiten der Selbstregulierung werden dem Erwachsenen in Krisen oder Belastungszeiten zur Verfügung stehen.

Mit der psychodynamischen Analyse lässt sich recht genau erkennen, auf welchem Niveau und in welcher Entwicklungsphase bestimmte Reifungsschritte gestört wurden [4]. Selbstverständlich entwickeln wir uns als Erwachsene weiter und können diese Defizite ausgleichen bzw. nachreifen.

\section{Neurobiologie und Trauma- forschung}

Die Neurobiologie hat revolutionierende Erkenntnisse für die Entwicklung des Gehirns durch wiederholtes Erleben von traumatisierenden Situationen, insbesondere im frühen Kindesalter, gewonnen.

In den ersten $1 \frac{1}{2}$ Jahren (frühe Bindungsphase) werden so viele Synapsen verknüpft wie in keiner späteren Lebensphase. In dieser frühen Reifungszeit ist unser Gehirn extrem beeindruckbar. Deshalb prägen psychische Belastungen unsere Seele in dieser Phase nachhaltig. Die neuroendokrinen Stressregelkreise (Hypothalamus-Hypophysen-NebennierenrindenRegelkreis) und damit viele vegetative Überreaktionen werden durch sich wiederholende Traumata sensibilisiert und es bahnen sich negative Stressmuster. Homöopathie kann diese vegetativen Sensationen ausgleichen. Das ist sehr wertvoll!

In dieser frühen Bindungsphase werden seelische Überlebensstrategien geprägt. Im Erwachsenenalter können sich dann unter Krisen und Belastungen unter 
anderem Depressionen, Angststörungen und Psychosen entwickeln.

Pathologische Gemütssymptome sind demnach als Verstärkung eines zugrundeliegenden psychischen Reaktionsmusters zu verstehen, das vor allem durch ein Zusammenwirken von angeborener Wesenheit, schädigender Erziehung und Umfeld geprägt wird bzw. die Folgen realer, nicht zu verarbeitender Traumata darstellt.

„Langfristig wird die ... Strukturierung des kindlichen Gehirns jedoch weniger durch das tatsächliche erlebte Trauma gefährdet, sondern durch die dadurch beim Kind ausgelöste Zerstörung von Sicherheit-bietenden emotionalen Bindungen, Selbstwertkonzepten und inneren Leitbildern. Wichtigstes Ziel aller therapeutischen Bemühungen muss es daher sein, Bedingungen zu schaffen, die es einem traumatisierten Kind ermöglichen, diese wichtigsten Ressourcen zur Bewältigung von Angst und Stress möglichst rasch wieder zurückzugewinnen.“ [2]

Psychische Störungen können transgenerational weitergegeben werden.

Schwer traumatisierte Eltern verhalten sich oft dissoziativ/kontaktlos. Sie geraten auch immer wieder in Zustände von panischer Hilflosigkeit und Ohnmacht und machen ihren Kindern damit Angst! Manche werden durch ihre Kinder unbewusst an ihr eigenes Trauma erinnert. Die Abhängigkeit und Hilflosigkeit besonders der kleinen Kinder, ihre Bindungswünsche, ihre Bedürftigkeit, ihr Weinen, ihr Kummer und Schmerz, aber auch ihre Ablösungswünsche oder Abgrenzungsversuche können die Eltern unbewusst in ihren eigenen Traumata antriggern. Gefühle von Wut, feindlichen Gefühlen gegenüber dem Kind oder Scham können bei den Eltern zu abrupten Handlungsabbrüchen oder Gewalt führen.

Eltern mit ängstigendem, ängstlichem oder hilflosem Verhalten können ihren Kindern keinen sicheren emotionalen Boden geben. Die Eltern verhalten sich für das Kind nicht vorhersehbar. Ein erschreckend hoher Anteil aller Kinder von schwer traumatisierten Eltern entwickelt ein solches traumatisiertes Bindungsmuster mit widersprüchlichen Verhaltensmustern und tranceartigen Zuständen.

Sehr empfehlenswert zu lesen sind hier Veröffentlichungen von Gerald Hüther [2] und Joachim Bauer [1].

\section{Fazit}

\section{Hereditäre Gemütskrankheiten}

Das Denkmodell der hereditären, also vererbten Gemütskrankheiten (wie von den Miasmatikern und auch von einigen Psychiatern vertreten) erscheint mir vor allem von der erläuterten Psychodynamik und Traumaforschung her betrachtet - als eine biologistische Arbeitshypothese, die sich zahlreichen kritischen Fragen stellen muss. Sie erklärt zum Beispiel nicht die starke Zunahme von schweren psychischen Erkrankungen, wie Depressionen, Angsterkrankungen, Zwängen und psychotischen Episoden ohne familiäre Häufung.

\section{Somatogene Ursache}

Die somatogene Erklärung halte ich für einen grundsätzlichen Denkfehler Hahnemanns. Psychische Symptome sind weder ein Palliativum für schwere körperliche Erkrankungen, noch eine Verschiebung organischer Erkrankungen in den Gemütsbereich. Eine Ausnahme bilden hier direkt einwirkende hirnschädigende Prozesse, die selbstverständlich zu Wesensveränderungen führen können.
Verschiebung auf die körperliche Ebene

Richtiger erscheint der Blickwinkel, dass ein psychischer Konflikt auf die körperliche Ebene verschoben wird. Die Auslastung der psychosomatischen Kliniken spricht für sich. Psychotherapeutisch wird es als beginnende Heilung angesehen, wenn die körperlichen Beschwerden sich verringern und das darunterliegende psychische Thema (z.B. sich verlassen oder nicht geliebt fühlen, Erinnerung an traumatische Erlebnisse) deutlich wird. Hier gibt es, wegen der fehlerhaften Grundannahme, Irritationen in der homöopathischen Verlaufsbewertung der Gemütssymptome. Wenn es dem Patienten körperlich besser geht, es ihm aber psychisch „nicht gut“ geht, wird dies als fehlerhafte Verschreibung missinterpretiert.

Fallbeispiel. Ich möchte ein kleines Beispiel einfügen, wie man die Selbstbeschreibung von Patienten nutzen kann, ohne interpretativ zu werden. Folgende Patientin war noch nie in Psychotherapie, ihre Aussagen sind deshalb „pur“ und nicht „manipuliert durch Therapeuten“, was ja manchmal ein unsinniger Vorwurf von Homöopathen ist.

Die Patientin kommt mit der Diagnose Reizmagen und Reizdarm in Behandlung. Medizinische Untersuchungen ergaben keine weiteren Auffälligkeiten. Breiiger bis durchfallartiger Stuhlgang, mehrfach am Tag, Bauchschmerzen (während des Essens oft krampfartig mit baldiger Entleerung), dazu Übelkeit als Dauerzustand.

Die zwingenden, schmerzhaften Darmentleerungen und die ständige Übelkeit erlebe sie als „unkontrolliertes Ausgeliefertsein“ an ihrem Arbeitsplatz. „Ich glaube, mir ist in den letzten Jahren einiges auf den Magen geschlagen.“ Die Beschwerden hätten sich verschlechtert nach einem Konflikt mit ihrer Chefin, von der sie sich „nicht wertgeschätzt und gedemütigt fühle“. Sie sei eine engagierte und belastbare Mitarbeiterin, die sich auch um das Wohl der anderen bemühe. „Ich hatte von früh an Sorge um andere, weil ich mit einer behinderten Schwester aufgewachsen bin. Ich wollte meiner Mutter keine Sorgen bereiten, ihr nicht auch noch zur Last fallen." Mit Anfang zwanzig erkrankte sie an Diabetes. Dies erschütterte sie schwer, weil sich „nun doch ihre Mutter in Sorge um sie stürzte“. 
Sie verschweigt ihre Erkrankung, weil sie meint, dass man sie für nicht belastbar und nicht leistungsfähig halten könnte. Sie treibt sich an und arbeitet weit über ihre Belastbarkeit hinaus. „Ich bin eigentlich nur durchs Leben gehetzt, ich lebe zwar, aber ich komme nicht vor. Innere Entspannung finde ich schwer. Und wenn ich zur Ruhe komme, werde ich traurig.“

Nach der Einnahme von Staphysagria C 200 (3 Globuli direkt auf die Zunge, dann 4 Tage verkleppert, morgens und abends 2 Schlucke) hört der Durchfall auf - sie wird unendlich traurig, weint und ist unruhig. Sie bekommt Angst vor dem Arbeitsalltag, „kann ich das alles schaffen?“. Homöopathisch betrachtet ist das Mittel treffend genug - es gibt nichts zu tun. Aber es ist ein heikler Punkt in der Patientenführung. Das körperliche Symptom ist erst einmal beschwichtigt und eines der zugrundeliegenden Gefühle ist da. Es muss von der Patientin auch autobiografisch verstanden und angenommen werden. Jetzt geht es um die emotionale Begleitung eines instabilen Zustands. Die Patientin braucht emotionale Entlastung, muss das Vertrauen in sich entwickeln, dass und wie sie krisenhafte Momente bewältigen kann. Sie muss z.B. lernen, für sich zu sorgen und Nein zu sagen. Das gibt Selbstvertrauen und nimmt den Druck, auf der körperlichen Ebene zu reagieren. Die Patientin entwickelt sich gut in diese Richtung. Die Darmentleerungen könne sie besser kontrollieren, während ihrer Arbeit sei ihr Darm nun ruhiger.

Intensive Träume, Weinen, innerliches Aufgelöstsein oder andere intensive Empfindungen sind auch psychische Heilungsreaktionen im Sinne von Gewahrwerden der inneren Befindlichkeit. Alte verdrängte oder verleugnete seelische Verletzungen können an die Oberfläche kommen, mit der heutigen Distanz verstanden, betrauert und „verdaut“ werden. Das Bewusstwerden der Wahrheit ist schmerzhaft oder traurig, aber letztlich auch beruhigend. Oftmals beschreiben die Patienten dies auch gleichzeitig als erleichternd, was die Mittelwahl bestätigt.

Generell ist es so: Wenn nach der Mitteleinnahme eine von dem Patienten als fremd empfundene Reaktion auftaucht (sowohl körperlich als auch psychisch), dann muss man die Mittelwahl sehr wohl überprüfen.

\section{Einseitige Krankheit}

Psychische Erkrankungen sind auch nicht als „einseitige Krankheit“ zu betrachten. Wie Sie sicherlich auch beobachtet haben, gibt es psychisch Kranke mit vielen körperlichen Beschwerden und andere, die vergleichsweise organisch gesund sind. Liegt die Schädigung im emotionalen Bereich, zeigen sich vor allem dort bestimmte, auffällige Verhaltensweisen. Sie können, müssen aber nicht körperliche Beschwerden nach sich ziehen.

\section{Schlussbetrachtung}

Samuel Hahnemann und Siegmund Freud waren große visionäre Denker und Wegbereiter ihrer Zeit. Auch die heutigen Psychotherapeuten haben Lehrsätze Freuds kritisch weiterentwickelt. Hahnemann hat sich nicht an konventionelles Denken gehalten. Ganz im Gegenteil: Er hat rigoros alte Denkstrukturen verlassen. In seinem Sinne dürfen wir uns von einigen seiner Arbeitshypothesen lösen, ohne ihn in seiner Großartigkeit aus den Augen zu verlieren.

\section{Online zu finden unter:}

http://dx.doi.org//10.1055/s-0033-1334398

\section{• Literatur}

[1] Bauer J. Das Gedächtnis des Körpers: Wie Beziehungen und Lebensstile unsere Gene steuern. 19. Aufl. München: Piper; 2004

[2] Hüther G. Die Folgen traumatischer Kindheitserinnerungen für die weitere Hirnentwicklung. Im Internet: http://www.agsp. de/html/a34.html; Stand: 01.07.2013

[3] Rudolf G. Psychotherapeutische Medizin und Psychosomatik. 4. Aufl. Stuttgart: Thieme; 2007

[4] Rudolf G. Strukturbezogene Psychotherapie: Leitfaden zur psychodynamischen Therapie struktureller Störungen. 2. Aufl. Stuttgart: Schattauer; 2006

[5] Venzlaff U, Dulz B, Sachsse U. Zur Geschichte der Psychotraumatologie. In: Sachsse U, Hrsg. Traumazentrierte Psychotherapie. Stuttgart: Schattauer; 2004

[6] www.wikipedia.de

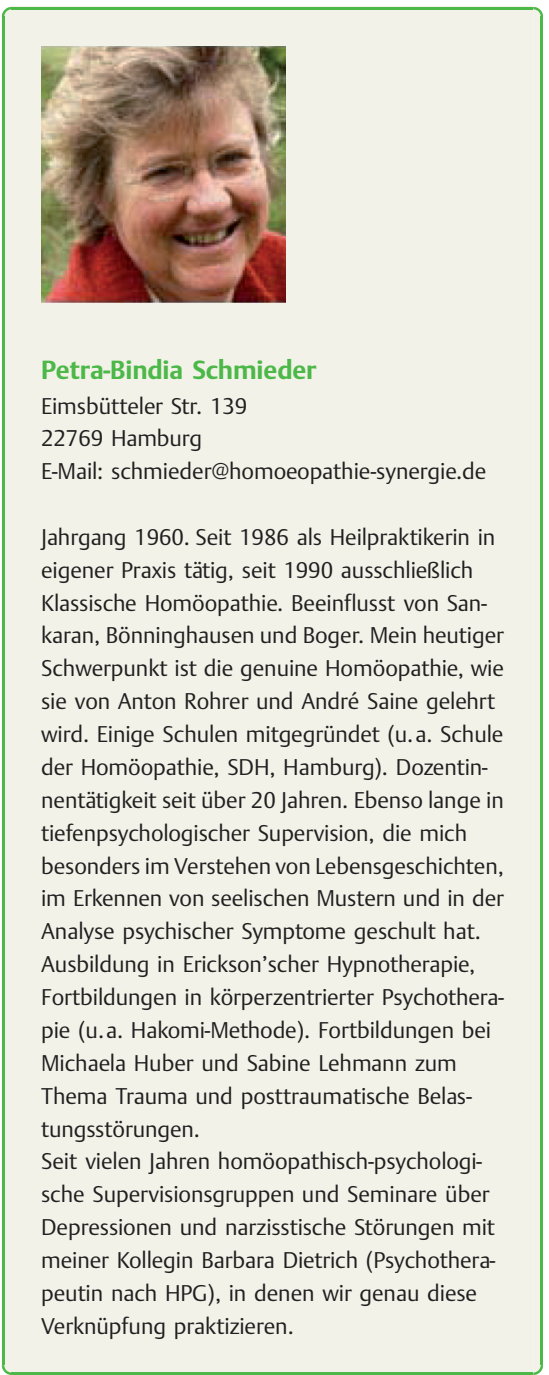

Petra-Bindia Schmieder

Eimsbütteler Str. 139

22769 Hamburs

E-Mail: schmieder@hom

Jahrgang 1960. Seit 1986 als Heilpraktikerin in eigener Praxis tätig, seit 1990 ausschließlich

Klassische Homöopathie. Beeinflusst von Sankaran, Bönninghausen und Boger. Mein heutiger Schwerpunkt ist die genuine Homöopathie, wie sie von Anton Rohrer und André Saine gelehrt wird. Einige Schulen mitgegründet (u.a. Schule Homöathie, SDH, Hamburg). Dozentinbesonders im Verstehen von Lebensgeschichten, Erkennen von seelischen Mustern und in der

isch-psychologivervisionsgruppen und Seminare über Depressionen und narzisstische Störungen mit veutin nach $\mathrm{HPG}$ ), in den
Verknüpfung praktizieren. 